\title{
SOME REMARIS ON THE FINITENESS CONDITIONS OF RINGS
}

\author{
AHMED A. M. KAMAL
}

\begin{abstract}
The aim of this paper is to study the finiteness of rings. We prove that if $A$ is a regular left-self-injective ring, then $A$ is of type III (purely infinite) implies that $E(A[x])$ is, and $A$ contains an abelian idempotent if and only if $E(A[x])$ contains an abelian idempotent. Also we prove that.

If $A$ is a regular left self-injective ring and $J$ is a left ideal in $A[x]$ such that $C(J)$ is an essential left ideal in $A$, then there exists a countably generated left ideal $J^{\prime}$ in $A[x]$ such that $C\left(J^{\prime}\right)$ is an essential left ideal in $A$, and if $J^{\prime}$ is an essential left ideal in $A[x]$, then $J$ is an essential left ideal in $A[x]$.
\end{abstract}

\section{Introduction}

As special case of the work in [6], we have that " $A$ regular left self-injective ring $A$ is of type $\mathbb{I}, \mathbb{I}_{f}, \mathbb{I}_{\infty}$ if and only if $E(A[x])$ is of the same respective type. In this paper we are interested in study the relation between the finiteness of the regular left self-injective rings and the finiteness of the injective envelope of their polynomial rings.

Throughout this paper all rings are associative with unit. $\mathrm{A}$ ring $A$ is regular provided that for every $x \in A$, there exists $y \in A$ such that $x y x=x$. $\mathbb{A}$ ring $A$ is unitregular provided that, for each $x \in A$, there is an invertable element $u \in A$ such that $x=x u x$. A regular ring is abelian provided all idempotents in $A$ are central. A ring $A$ is called left self-injective if ${ }_{A} A$ is injective $A$-module. A module $M$ is directly finite if $M$ is not isomorphic to any proper direct summand of itself and a ring $A$ is directly finite if $A A$ is directly finite $A$-module and it is directly infinite if it is not directly finite. An idempotent $e$ in a ring $A$ is called faithful in $A$ if 0 is the only central idempotent of $A$ which is orthogonal to e. An idempotent, $e$ in a regular $\operatorname{ring} A$ is called abelian (directly finite) idempotent if $e A e$ is abelian regular (directly finite) ring. A regular, left self-injective ring is said to be purely infinite if it contains no nonzero directly finite central idempotent. And it is said to be of type II if it contains a faithful directly finite idempotent but it contains no nonzero abelian idempotents. Moreover it is called of type $\mathrm{II}_{f}\left(\mathrm{II}_{\infty}\right)$ if it is of type II and directly finite (purely infinite). And it is called of type III if it contains no nonzero directly finite idempotents. 
A ring $A$ is said to be a Baer ring if every left (right) annihilator is of the form $A e(e A), e$ is an idempotent in $A$. The central cover of an element $x \in A$, written $C(x)$, is the smallest central idempotent $e$ in $A$ satisfying $e x=x$. By the injective invelope of a ring $A$ we mean the injective envelope of it as a left module over itself and we denoted it by $E(A)$. Finally the set of all central idempotents in a $\operatorname{ring} A$ is denoted by $B(A)$.

\section{Preliminaries}

In this section we collect some results and a consequences of them, will be needed in this paper.

(2.1) Let $A$ be a Baer ring, $e$ be an idempotent in $A$ and $f$ be an idempotent in $e A e$. If $f$ is a directly finite idempotent in $e A e$, then $f$ is a directly finite idempotent in $A$.

(2.2) Let $e, f$ be idempotents in a Baer ring $A$. Then the following conditions are equivalent

(i) $A e$ is isomorphic to $A f$ as a left $A$-modules

(ii) $e A$ is isomorphic to $f A$ as a right $A$-modules

(iii) There exist elements $x$ and $y$ such that $x$ e Af, $y$ f $A e, x y=e$ and $y x=f$

Two idempotent $e$ and $f$ in a Baer ring $A$ are called equivalent and denoted by $e \sim f$ if they satisfy the equivalence conditions (2.2).

(2.3) A Baer ring $A$ is directly finite if and only if for every idempotent $e \in A, e \sim l$ implies $e=$ l.

(2.4) Let $A$ be a Baer ring with no nonzero nilpotent ideals. If $e=e^{2} \in A$ and $f \in B(e A e)$, then $C(f) e=f$

The proof of 2.1, 2.2, 2.3 and 2.4 can be found in [8]

Notice that a regular ring is a Baer ring if and only if the lattice of principal left (ring) ideals of it is complete and hence every regular left (ring) self-injective ring is a Baer ring. Therefore (2.1), (2.2), (2.3) and (2.4) are satisfied for regular left self-injective rings.

(2.5) Let $A$ be a left nonsingular ring, $N$ be a submodule of the left $A$-module $M$. Then $N$ is essential in $M(N \leq e M)$ if and only if $N x^{-1}$ is an essential left ideal in $A$ for each $x \in M$, where $N x^{-1}=\{a \in A: a x \in N\}$.

The proof of (2.5) can be in [5].

A consequence of (2.5) is that if $I$ is essential left ideal of a left nonsingular $\operatorname{ring} A$, then for each $b \in A$, there exists an essential left ideal $K$ of $A$ such that $0 \neq K b \subset I$.

(2.6) Let $A$ be a regular, left self-injective ring, and let $\left\{J_{i}\right\}$ be an independent family of left ideals of $A$. Then there exist orthogonal idempotents $e_{i} \in A$ such that each $J_{i} \leq{ }_{, e} A e_{i}$. If the $J_{i}$ are also principal, then each $J_{i}=A e_{i}$.

The proof of (2.6) can be found in [2].

Let $A$ be a regular left self-injective ring and $I$ be a left ideal in $A$. If $\left\{K_{i}\right\}$ is a maximal independent family of principal left ideals of $A$ such that $K_{i} \subset I$ for each $i$, 
then $\oplus K_{i} \leq_{e} I$. By using (2.6) we can find an orthogonal idempotents $\left\{e_{i}\right\}$ such that $A e_{i}=K_{i}$ for each $i$. Therefore $\oplus A e_{i} \leq e_{e} I$. On the other hand $E\left({ }_{A} I\right)$ is a direct summand in $A$ which implies the existance of an idempotent $e \in A$ such that $I \leq{ }_{e} A e$ Moreover $e$ is unique.

(2.7) Every regular, left self-injective ring satisfies general comparability.

The proof of (2.7) can be found in [9]

(2.8) If $A$ is a unit-regular ring, then every finitely generated projective $A$-module is directly finite, consequently, $M_{n}(A)$ is directly finite for all $n$

The proof of (2.8) can be found in [4].

(2.9) If $A$ is a directly finite regular ring satisfying general comparability, then $A$ is unit-regular.

The proof of (2.9) can be found in [10].

From (2.7) and (2.9) a directly finite regular left self-injective ring is a unit-regular. Conversely if $A$ is a unit-regular left self-injective, then ${ }_{A} A$ is a finitely generated nonsingular left $A$-module, which implies that ${ }_{A} A$ is projective, hence (2.8) shows that $A$ is a directly finite.

(2.10) Let $A$ be a semiprime, left nonsingular ring and $e$ be an idempotent of $A$. Then e $E(A) e$ is the injective envelope of $e A e$

The proof of 2.10 can be found in [1]

(2.11) Let $A$ be a regular, left self-injective ring. If $A$ is directly finite, then every nonzero ideal of $A$ contains a nonzero central idempotent.

(2.12) For a regular left self-injective $\operatorname{ring} A$ the following conditions are equivalent.

(i) $A$ is purely infinite

(ii) $n A^{A} \lesssim A^{A}$ for some integer $n \geq 2$

(iii) $n A^{A} \simeq A^{A}$ for all positive integer $n$

(iv) $E\left(\chi_{0} A^{A}\right) \simeq A^{A}$

The proof of (2.11) and (2.12) can be found in [9]

(2.13) Let $A$ be a regular left self-injective ring, $\sigma \in \operatorname{Aut}(A)$ and $D$ be a $\sigma$-derivation of $A$. Then $B(E(A[X, \sigma, D]))=(B(A))^{\sigma}$, where $(B(A))^{\sigma}=\{e \in B(A): \sigma(e)=e\}$.

(2.14) If $A$ is an abelian regular ring, then $E(A[X, D])$ is an abelian regular ring.

The proof of (2.13) and (2.14) can be found in [6].

\section{Finiteness conditions of rings}

Notice first that, if $A$ is a left nonsingular ring, then $A[x]$ is left nonsingular and hence $E(A[X])$ is a regular left self-injective ring.

Proposition 3.1. If $A$ is a regular left self-injective ring of type $I I$, then $E(A[X])$ is of type III.

Proof. Assume that $E(A[X])$ is not of type $I I I$ hence there exists a non zero directly finite idempotent $f$ in $E(A[X])$, which implies that $E(A[X]) f$ is directly finite 
and $I=I=E(A[X]) f \cap A[X]$ is a non zero left ideal in $A[X]$, since $A$ is a regular ring, then there exists a non zero polynomial $p$ in $I$ with minimal longth and $p=e X^{n}+$ $a_{n-1} X^{n-1}+\cdots+a_{0}$. Moreover $p=e p=e X^{n}+e a_{n-1} X^{n-1}+\cdots+a_{0}, e=e^{2} \in A$ we define

$$
\begin{aligned}
\varphi: A[X] & \rightarrow A[X] P \\
q & \rightarrow q \cdot P
\end{aligned}
$$

It is clear that $\varphi$ is an epimorphism with $\operatorname{Ker} \varphi=\operatorname{Ann}(P)=A[X](1-e)$, which implies that $A[X] P \simeq A[X] e$, hence $E(A[X]) e \simeq E(A[X] P) \subseteq E(A[X]) f$, which implies that $E(A[X]) e$ is directly finite, therefore $A e$ is directly finite and hence $e$ is a directly finite idempotent in $A$ which is a contradiction.

Proposition 3.2. Let $A$ be a regular left self-injective ring. If $A$ is purely infinite, then $E(A[X])$ is purely infinite.

Proof. From (2.13), we have $B(E(A[X]))=B(A)$, and hence the proof of the proposition is clear.

Recal that if $A$ is a Baer ring and $e=e^{2} \in A$ is a nonzero idempotent, then $A$ is of type $I I I$ implies that $e A e$ is of type $I I I$ (see [8]). Therefore this result is also true for
regular left self-injective rings.

Example 3.3. This example shows that the previous result is not true for purely infinite regular left self-injective rings. Let $K$ be a field and $A=\operatorname{End}_{K}\left(K^{N}\right)$, then $A$ is a purely infinite regular left self-injective ring. We define $P_{0}: K^{N} \rightarrow K, P_{0}$ is a projection and $P_{0} A P_{0} \simeq K$ is directly finite.

Proposition 3.4. Let $A$ be a regular left self-injective ring and $f$ be a nonzero central idempotent in $A$. If $A$ is purely infinite, then $f A f$ is purely infinite.

Proof. Since $A$ is purely infinite ring, then (2.12) implies that $A \simeq \mathbb{E}\left(\oplus_{i} A e_{i}\right)$ with $A \simeq A e_{i}$ for each $i \in N$ and the $e_{i}^{\prime^{*}}$ are idempotents in $A$. By using (2.2), we have that, for each $i \in N$, there exist $x_{i} \in A e_{i}, y_{i} \in e_{i} A$ such that $x_{i} y_{i}=1$ and $y_{i} x_{i}=e_{i}$. Which implies that $\left(f x_{i} f\right)\left(f y_{i} f\right)=f,\left(f y_{i} f\right)\left(f x_{i} f\right)=e_{i} f$ with $f x f \in f A e_{i} f=A e_{i} f$ and $f y f \in e_{i} f A$, hence $A f \simeq A e_{i} f$ for each $i \in N$. Since $A$ is injective, there exists an idempotent $h$ in $A$ such that $E\left(\oplus_{i} A e_{i}\right) \simeq A h$ hence $A \simeq A h$, which implies that $A f \simeq A h f$ therefore $A f \simeq E\left(\oplus_{i} A e_{i} f\right)$.

Condition * We say that a ring $A$ satisfies condition * if for each sequence $e_{1}, e_{2}, \ldots$, $e_{n}, \ldots$ of nonzero idempotents in $A$, there exists $k \in N$ such that $C\left(e_{k}\right) \in e_{k-1} A e_{k-1}$,
where $C\left(e_{k}\right)$ is the central cover of $e_{k}$ in $A$.

Notice that, every division ring satisfies condition *. Also there exists a regular left self-injective ring which does not satisfy condition * as the following example shows. Example 3.5. Let $K$ be a field and $A=\operatorname{End}_{k}\left(K^{N}\right)$ then $A$ is a regular left
self-injective ring. 
Let $\left\{f_{n}: n \in N\right\}$ be a base of $K^{N}$ as a vector space over $K$, we define $V_{0}=K^{N}, V_{1}=$ $\oplus_{n \in N}\left(f_{2 n} K\right), V_{2}=\oplus_{n \in N}\left(f_{2^{2} n} K\right), \ldots, V_{p}=\oplus_{n \in N}\left(f_{2^{p} n} K\right)$, then $V_{0} \supseteq V_{1} \supseteq V_{2} \supseteq$ $\ldots V_{n} \supseteq \ldots$, let $P_{n}: V_{0} \rightarrow V_{n}$ be the projections from $V_{0}$ into $V_{n}$ for each $n \in N$, then $P_{1}, P_{2}, \ldots, P_{n}, \ldots$ is a sequence of idempotents in $A$ and $\{0,1\}$ is the set of all central idempotents in $A$.

Proposition 3.6. If $A$ is a regular left self-injective ring such that $E(A[X])$ satisfies condition *, then $A$ is purely infinite implies that $E(A[X])$ is of type III.

Proof. Assume that $E(A[X])$ is not of type $I I I$, hence $E(A[X])$ contains a directly finite idempotent say $e_{1}$. Therefore (2.11) implies that every nonzero ideal in $e_{1} E(A[X]) e_{1}$ contains a nonzero central idempotent in $e_{1} E(A[X]) e_{1}$. Since every regular ring is a semiprime, then by using (2.4), there exists a central idempotent $e_{2}$ in $e_{1} E(A[X]) e_{1}$ such that $C\left(e_{2}\right) e_{1}=e_{2}$, hence $e_{2} E(A[X]) e_{2}=e_{1} C\left(e_{2}\right) E(A[X]) C\left(e_{2}\right) e_{1}$ $\subseteq e_{1} E(A[X]) e_{1}$. Therefore $e_{2} E(A[X]) e_{2}$ is a directly finite regular left self-injective ring, which implies the existance of an idempotent $e_{3} \in e_{2} E(A[X]) e_{2}$ which is central in $e_{2} E(A[X]) e_{2}$ and directly finite in $E(A[X])$. And so on, we obtain a sequence of idempotents $e_{1}, e_{2}, \ldots, e_{n}, \ldots$ in $E(A[X])$ such that, each $e_{i}$ is directly finite in $E(A[X])$ and each $e_{i}$ is central in $e_{i-1} E(A[X]) e_{i-1}$. Therefore there exists $e_{k}$ for some $k \in N$, such that $C\left(e_{k}\right)$ is contained in $e_{k-1} E(A[X]) e_{k-1}$ and $e_{k}=C\left(e_{k}\right) e_{k-1}$. Moreover $C\left(e_{k}\right)\left(e_{k-1} E(A[X]) e_{k-1}\right) C\left(e_{k}\right)=e_{k} E(A[X]) e_{k}$, which implies that $C\left(e_{k}\right)$ is a directly finite idempotent in $e_{k-1} E(A[X]) e_{k-1}$. Therefore (2.4) implies that $C\left(e_{k}\right)$ is a central directly finite idempotent in $E(A[X])$, moreover (2.13) implies that $C\left(e_{k}\right)$ is a central directly finite idempoten in $A$ which is a contradiction.

Remark 3.7. The proof of proposition 3.6 shows that, if $E(A[X])$ satisfies the hypothesis of the proposition, then $E(A[X])$ contains directly finite idempotent, implies that it contains a central directly finite idempotent.

Notice that for unit-regular ring, the statement $A$ is directly finite implies that $E(A)$ is directly finite is not always true as the following example shows.

Example 3.8. Let $K$ be a field, then $M_{2^{n}}(K)$ is a regular directly finite ring for each $n \in N$. For each $n \in N$, we identify for each element $X$ in $M_{2^{n}}(K)$, the element $\left(\begin{array}{cc}X & 0 \\ 0 & X\end{array}\right)$ in $M_{2^{n+1}}(K)$ and hence $M_{2^{n}}(K) \subseteq M_{2^{n+1}}(K) \subseteq \cdots$ for each $n \in N$. Since the direct limit of regular rings is a regular ring, and also the direct limit of directly finite rings is a directly finite ring, then $A=\lim _{\rightarrow} M_{2^{n}}(K)$ is a regular directly finite ring. But the left injective envelope of $A$ and the right injective envelope of $A$ are different, both of which are of type III (see [3] theorem II.3.1) Therefore $E(A)$ is not directly finite.

Proposition 3.9. If $A$ is a left non singular ring such that $M_{n}(A)$ is directly infinite for some $n \in N, n \geq 2$, then $E(A)$ is a directly infinite.

Proof. Since $A$ is left nonsingular, then the maximal left quotient $\operatorname{ring} Q(A)$ is regular left self-injective ring. If $E(A)$ is directly finite, then $Q(A)$ is also directly finite 
and hence unit-regular. Therefore (2.8) implies that $M_{n}(Q(A))$ is directly finite for each $n \in N, n \geq 2$, whence $M_{n}(A)$ is directly finite for each $n \in N, n \geq 2$, which is a contradiction.

If $A=\lim M_{2^{n}}(E)$ as in example 3.8 , then $A[X]$ is directly finite, but $E(A[X])$ is not directly finite. We discuss now that, if $A$ is directly finite regular left self-injective ring, is $E(A[X])$ is directly finite.

Theorem 3.10. If $A$ is a nonsingular ring, then $E(A[X])$ is directly infinite if and only if there exists left ideals $I$ and $J$ in $A[X]$ such that $I \simeq J, I \leq_{e} A[X]$ and $J \mathbb{L}_{e} A[X]$ as left $A[X]$-modules.

Proof. Notice first that $E(A[X])$ is a Baer ring

(1) Assume that, there exist left ideals $I, J$ in $A[X]$ such that $I \simeq J, I \leq_{e} A[X]$ and $J \not L_{e} A[X]$ as left $A[X]$-modules. Hence $E(I)$ and $E(J)$ are direct summands of $E(A[X])$, moreover $I \leq_{e} A[X]$ implies that $E(I)=E(A[X])$, also $E(J)=E(A[x]) f$ for some nonzero idempotent $f$ in $E(A[X])$ with $f \neq 1$. But $I \simeq J$ implies that $E(I) \simeq E(J)$, hence $f \sim 1$. Therefore (2.3) shows that $E(A[X])$ is a directly infinite ring.

(2) Assume that $E(A[X])=B$ is directly infinite then there exists idempotent $f$ in $B$ such that $f \sim 1$ and $0 \neq f \neq 1$. Therefore there exists an isomorphism $\psi: B f \rightarrow B$ of left $B$-modules. We have that $B f \cap A[X] \leq_{e} B f$ as left $A[X]$-modules, since $\psi$ is an isomorphism, $\psi(B f \cap A[X]) \leq_{e} B$, which implies that

$$
\psi(B f \cap A[x]) \cap A[X] \leq_{e} A[X]
$$

Also we have that $\psi(B f \cap A[X]) \cap A[x] \leq_{e} B$. Since $\psi^{-1}$ is an isomorphism, then

$$
J=\psi^{-1}\left(\psi(B f \cap A[X] \cap A[X]) \leq_{e} B f .\right.
$$

Now

$$
\begin{aligned}
J & \subseteq \psi^{-1}(\psi(B f \cap A[x])) \cap \psi^{-1}(A[x]) \\
& \subseteq(B f \cap A[X]) \cap B f \\
& \subseteq A[X]
\end{aligned}
$$

also $J \cap(B(1-f) \cap A[X]) \subseteq(B f \cap A[X]) \cap(B(1-f) \cap A[X]) \subseteq B f \cap B(1-f)=0$, therefore $J$ is not an essential left ideal in $A[X]$, conversly, from (*) we have that $I=$ $\psi(J)=\psi(B f \cap A[x]) \cap A[X] \leq_{e} A[X]$, also we have $J \stackrel{\psi / J}{\longrightarrow} I=\psi(J)$ is an isomorphism of left $A[X]$ - modules.

Remarks 3.11. As a special case of [7], if $I$ is a left (right, two sided) ideal in $A[X]$, then $C_{n}(I)=\left\{0 \neq a \in A: \exists P=a X^{n}+a_{n-1} \cdot X^{n-1}+\cdots+a_{0}, P \in I\right\} U\{0\}$ is a left (right, two sided) ideal in $A$ for each $n \in N$. Moreover $C_{n}(I) \subset C_{n+1}(I)$ for each $n \in N$, which implies that $C(I)=\cup_{n \in N}, C_{n}(I)$ is a left (right, two sided) ideal in $A$. Notice that, if $I$ is an essential left (right, two sided) ideal in $A[X]$, then $C_{n}(I)$ so is. But if $A$ is a nonsinguar ring and $I$ is a eft (right) ideal in $A[X]$ such that $C_{0}(I)$ is an essential 
in $A$, then $I$ is an essential in $A[X]$. Moreover the converse is not true. Finally, if $A$ is a semiprime ring and $I$ is a two sided ideal in $A[X]$, then $I$ is an essential in $A[X]$ if and only if $C[I]$ is an essential in $A$.

Now let $A$ be a regular left seft-injective ring and $J$ be a left ideal in $A[X]$ such that $C(J)=\cup_{n \in N} C_{n}(J) \leq_{e} A$

We shall construct a left ideal $J^{*}$ in $A[X]$ such that $J^{*} \subseteq J$ as follows. Since $C_{0}(J)$ is a left ideal in $A$, there exists an idempotent $e_{0}$ and orthogonal idempotents $\left\{f_{i_{0}}\right\}_{i_{0} \in I_{0}}$ in $A$ such that $\oplus_{i_{0} \in I_{0}} A f_{i_{0}} \leq_{e} C_{0}(J) \leq_{e} A e_{0}$. Also since $C_{0}(J) \subseteq C_{1}(J)$, there exists an idempotent $e_{1}$ and orthogonal idempotents $\left\{f_{i_{1}}\right\}_{i_{1} \in I_{1}^{\prime}}$ in $A$ such that $I_{1}^{\prime} \cap I_{0}=\phi$ and

$$
\left(\underset{i_{0} \in I_{0}}{\oplus} A f_{i_{0}}\right) \oplus\left(\underset{i_{1} \in I_{1}^{\prime}}{\oplus} A f_{i_{1}}\right) \leq_{e} C_{1}(J) \leq_{e} A e_{1}
$$

Similarly $C_{0}(J) \subseteq C_{1}(J) \subseteq C_{2}(J) \subseteq \cdots \subseteq C_{n}(J) \subseteq \cdots$ implies that $\left(\oplus_{i_{0} \in I_{0}} A f_{i_{0}}\right) \oplus$ $\left(\oplus_{i_{1} \in I_{1}^{\prime}} A f_{i_{1}}\right) \oplus \cdots \oplus\left(\oplus_{i_{n} \in I_{n}^{\prime}} A f_{i_{n}}\right) \leq_{e} C_{n}(J) \leq_{e} A e_{n}$ for each $n$. Note also that $I_{0} \subseteq$ $I_{1} \subseteq I_{2} \subseteq \cdots \subseteq I_{n} \subseteq \cdots$ where $I_{n+1}=I_{n} \cup I_{n+1}^{\prime}$ and $I_{n} \cap I_{n+1}^{\prime}=\phi, n=0,1,2, \cdots$

For each $i \in \cup_{n} I_{n}$, let $m=\min \left\{k \in N: i \in I_{k}^{\prime}\right\}$ hence $i=I_{m}^{\prime} \in I_{m}$, implies that $f_{i}=f_{i_{m}} \in C_{m}(J)$, therefore there exists $P=f_{i} X^{m}+a_{m-1} X^{m-1}+\cdots+a_{0} \in J$, and we take $P_{i}=f_{i} P \in J$. Now we define the left ideal $J^{*}$ as follows

$$
J^{*}=\sum_{i \in \bigcup_{n} I_{n}} A[X] P_{i}
$$

Proposition 3.12. $J^{*}$ is an essential left ideal in $J$.

Proof. To prove that, let for each $p \in N, J_{p}$ be that left ideal of $A[X]$ generated by the polynomials of $J$ of degree $\leq p$ and $J_{p}^{*}$ be the left ideal of $A[X]$ generated by the polynomials $P_{i}$ constructed above with degree $\leq p$.

First. we shall prove that $J_{0}^{*} \leq_{e} J_{0}$ Notice that $J_{0}=A[X] C_{0}(J)$ and $J_{0}^{*}=\oplus_{i_{0} \in I_{0}} A[X] f_{i_{0}}$. Let $f \in J_{0}$, then $f=b_{n} X^{n}+$ $b_{n-1} X^{n-1}+\cdots+b_{0}$ with $0 \neq b_{i} \in C_{0}(J), i=0,1, \cdots, n$, since $\oplus_{i_{0} \in I_{0}} A f_{i_{0}} \leq_{e} C_{0}(J)$ then for each $b_{i}$, there exists an essential left ideal $K_{i}$ of $A$ such that $0 \neq K_{i} b_{i} \subseteq$ $\oplus_{i_{0} \in I_{0}} A f_{i_{0}}$. Since $K=\cap_{i=0}^{n} K_{i} \leq_{e} A$ and $A$ is left and right nonsongular ring, then $0 \neq K b_{i} \subseteq \oplus_{i_{0} \in I_{0}} A f_{i_{0}}$ for each $i=0,1, \cdots, n$. Let $0 \neq \xi_{i} b_{i} \in \oplus_{i_{0} \in I_{0}} A f_{i_{0}}, \xi_{i} \in K$ for each $i=0,1, \cdots, n$, which implies that $0 \neq \xi_{j} f=\sum_{i=0}^{n} \xi_{j} b_{i} X^{i} \in \oplus_{i_{0} \in I_{0}} A[X] f_{i_{0}}=J_{0}^{*}$ for each $j=0,1, \cdots, n$. Therefore $J_{0}^{*} \leq_{e} J_{0}$.

NOw we shall prove that $J^{*} \leq_{e} J$. Let $Q \in J$, we use the induction on the degree of $Q$. If degree $Q=0$, then $Q=a \in C_{0}(J) \subseteq J_{0}$, since $J_{0}^{*} \leq_{e} J_{0}$, we can find an essential left ideal $K$ of $A[X]$ such that $0 \neq K a=K Q \subseteq J_{0}^{*} \subseteq J^{*}$.

Suppose that this is true for any polynomial $Q$ in $\bar{J}$ with degree less than or equal to $n-1$. Let $Q=a_{n} X^{n}+a_{n-1} X^{n-1}+\cdots+a_{0} \in J$ with $a_{n} \neq 0$, hence $a_{n} \in C_{n}(J)$. Let $p_{0}=\inf \left\{p \in N: a_{n} \in C_{p}(J)\right\}$, since $\oplus_{i_{j}} A f_{i_{j}} \leq_{e} C_{p_{0}}(J)$, where $i_{j} \in \cup_{\ell=0}^{p_{0}} I_{\ell}$, hence there 
exists $\lambda \in A$ such that $0 \neq \lambda a_{n} \in \oplus_{i_{j}} A f_{i_{j}}, i_{j} \in \cup_{\ell=0}^{p_{0}} I_{\ell}$ Therefore $\lambda a_{n}=\sum_{j=0}^{k} a_{i_{j}}^{*} f_{i_{j}}$, where $i_{j} \in \cup_{\ell=0}^{p_{0}} I_{\ell}$, and we can assume that $i_{0}<i_{1}<i_{2} \cdots<i_{k}<p_{0}$. Since $P_{i_{j}}=$ $f_{i_{j}} X^{i_{j}}+f_{i_{j}} a_{i_{j-1}} X^{i_{j}-1}+\cdots f_{i_{j}} a_{0} \in J^{*}$ with $P_{i_{j}}=f_{i_{j}} P_{i_{j}}$, hence $a_{i_{j}}^{*} P_{i_{j}} \in J^{*}$ which implies that $Q^{\prime}=a_{i_{k}}^{*} P_{i_{k}}+a_{i_{k-1}}^{*} X^{i_{k}-i_{k-1}} P_{i_{k-1}}+\cdots+a_{i_{0}}^{*} X^{i_{k}-i_{0}} P_{i_{0}}$ is in $J^{*}$ with leading coefficient $\sum_{j=0}^{k} a_{i_{j}}^{*} f_{i_{j}}$, where $i_{j} \in \cup_{\ell=0}^{p_{0}} I_{\ell}$. Therefore $Q_{1}=\lambda Q-X^{n-i_{k}} Q^{\prime}$ is of degree less than or equal to $n-1$, which implies the existance of an essential left ideal $L$ of $A[X]$ such that $L Q^{\prime} \subseteq J^{*}$, hence $L \lambda Q \subseteq J^{*}$. Let $K$ be the complement of $\ell_{A[X]}(\lambda Q)$ in $A[X]$, since $A[X]$ is left and right nonsingular (See $[6]$ lemma 3.1 ) then $\ell_{A[X]}(\lambda Q)$ is not essential in $A[X]$. Therefore $K$ is a nonzero left ideal in $A[X]$, which implies that $L \cap K \neq 0$, hence there exists $0 \neq g \in L \cap K$ such that $0 \neq g \lambda Q \in L \lambda Q$. Therefore $0 \neq L \lambda Q \subseteq J^{*}$ which implies that $J^{*}$ is an essential in $J$. follows.

Now we shall define other left ideal $J^{\prime}$ in $A[X]$ such that $J^{*}$ is an essential in $J^{\prime}$ as

We have that $\oplus_{i_{0} \in I_{0}} A f_{i_{0}} \leq_{e} C_{0}(J) \leq_{e} A e_{0}$, and since $\ell_{A}\left(e_{0}\right)=A\left(1-e_{0}\right)$, then $\left(\oplus_{i_{0} \in I_{0}} A f_{i_{0}}\right) \oplus \ell_{A}\left(e_{0}\right) \leq_{e} A$ and we define a homomorphism of left $A$-modules

$$
\left.\varphi: \underset{i_{0} \in I_{0}}{\oplus} A f_{i_{0}}\right) \oplus \ell_{A}\left(e_{0}\right) \rightarrow A
$$

such that $\varphi$ is the identity on $\oplus_{i_{0} \in I_{0}} A f_{i_{0}}$ and zero on $\ell_{A}\left(e_{0}\right)$, since $A$ is a left selfinjective, $\varphi$ can be extended to a homomrphism $\varphi^{*}: A \rightarrow A$. Let $f_{0}=\varphi^{*}(1)$ and put
$P_{0}=f_{0}$.

For each $i_{1} \in I_{1}^{\prime}, P_{i_{1}}=f_{i_{1}} X+f_{i_{1}} a_{i_{1}}^{0}$, we define two homomorphisms of left $A$-modules

$$
\varphi \text { and } \psi:\left(\underset{i_{0} \in I_{0}}{\oplus} A f_{i_{0}}\right) \oplus\left(\underset{i_{1} \in I_{1}^{\prime}}{\oplus} A f_{i_{1}}\right) \oplus \ell_{A}\left(e_{1}\right) \rightarrow A
$$

such that $\varphi$ is the identity on $\oplus_{i_{1} \in I_{1}} A f_{i_{1}}$ and zero otherwise, but $\psi\left(f_{i_{1}}\right)=a_{i_{1}}^{0}$ for each $i_{1} \in I_{1}$ and zero on $\left(\oplus_{i_{0} \in I_{0}} A f_{i_{0}}\right) \oplus \ell_{A}\left(e_{1}\right)$,similarly $\varphi$ and $\psi$ are extended to homomorphisms $\varphi^{*}$ and $\psi^{*}$, we let $f_{1}=\varphi^{*}(1), a_{1}^{0}=\psi^{*}(1)$ and define $P_{1}=f_{1} X+f_{1} a_{1}^{0}$. In general for each $i_{n} \in I_{n}^{\prime}$ we have that $P_{i_{n}}=f_{i_{n}} X^{n}+f_{i_{n}} a_{i_{n}}^{n-1} X^{n-1}+\cdots+f_{i_{n}} a_{i_{n}}^{0}$ we define homomorphisms of left $A$-modules $\varphi_{i}:\left(\oplus_{i_{0} \in I_{0}} A f_{i_{0}}\right) \oplus\left(\oplus_{i_{1} \in I_{1}^{\prime}} A f_{i_{1}}\right) \oplus \cdots \oplus$ $\left(\oplus_{i_{n} \in I_{n}^{\prime}} A f_{i_{n}}\right) \oplus \ell_{A}\left(e_{n}\right) \rightarrow A, i=0,1, \cdots, n$ as follows $\varphi_{n}$ is the identity homomorphisms at $\oplus_{i_{n} \in I_{n}} A f_{i_{n}}$ and zero otherwise, but $\varphi_{j}\left(f_{i_{n}}\right)=a_{i_{n}}^{j}$ for each $f_{i_{n}}$ and for $j=0,1, \cdots, n-$ 1 and zero otherwise $\varphi_{n}$ and $\varphi_{j}, j=0,1, \cdots, n-1$ can be extended to homomorphism $\varphi_{n}^{*}$ and $\varphi_{j}^{*}, j=0,1, \cdots, n-1$ from $A$ into $A$, we define $\varphi_{j}^{*}(1)=a_{n}^{j}, j=0, \cdots, n-1, \varphi_{n}^{*}(1)=$ $f_{n}$, also we define $P_{n}$ as the following $P_{n}=f_{n} X^{n}+f_{n} a_{n}^{n-1} X^{n-1}+\cdots+f_{n} a_{n}^{1} X+f_{n} a_{n}^{0}$ and we define $J^{\prime}=\sum_{n \in N} A[X] P_{n}$, which is countably generated left ideal in $A[X]$.

Remarks 3.13. Notice that $f_{i_{n}} f_{n}=f_{i_{n}} \varphi_{n}^{*}(1)=\varphi_{n}\left(f_{i_{n}}\right)=f_{i_{n}}$ and $f_{i_{n}} a_{n}^{j}=$ $f_{i_{n}} \varphi_{j}^{*}(1)=\varphi_{j}\left(f_{i_{n}}\right)=a_{i_{n}}^{j}$ for each $j=0,1, \cdots, n-1$, wich implies that $f_{i_{n}} P_{n}=P_{i_{n}}$ for

each $n \in N$ and since $J^{*}=\sum_{i_{j} \in \cup_{n} I_{n}} A[X] P_{i_{j}}$, we obtain that $J^{*} \subseteq J^{\prime}$.
(2) It is clear that $C\left(J^{\prime}\right)=\cup_{n \in N} C_{n}\left(J^{\prime}\right)=\sum_{n \in N} A f_{n}$ also $f_{i_{n}}=f_{i_{n}} \cdot f_{n}$, implies that
$\oplus_{i_{n} \in \cup_{n} I_{n}} A f_{i_{n}} \subseteq C\left(J^{\prime}\right)$. $\oplus_{i_{n} \in \cup_{n} I_{n}} A f_{i_{n}} \subseteq C\left(J^{\prime}\right)$. 
(3) $\oplus_{i_{n} \in \cup_{n} I_{n}} A f_{i_{n}} \leq_{e} C(J) \leq_{e} A$ and $\oplus_{i_{n} \in \cup_{n} I_{n}} A f_{i_{n}} \subseteq C\left(J^{\prime}\right) \subseteq A$ implies $C\left(J^{\prime}\right) \leq_{e} A$ and $\oplus_{i_{n} \in \mathrm{U}_{n} I_{n}} A f_{i_{n}} \leq_{e} C\left(J^{\prime}\right)$.

Propsition 3.14. $J^{*}$ is an essential left ideal in $J^{\prime}$.

Proof. Let $Q=\sum_{j-1}^{k} Q_{j} P_{t_{j}} \in J^{\prime}$, where $P_{t_{j}}=f_{t_{j}} X^{t_{j}}+f_{t_{j}} a_{t_{j}}^{t_{j}-1} X^{t_{j}-1}+\cdots+$ $f_{t_{j}} a_{i_{j}}^{1} X+f_{t_{j}} a_{i_{j}}^{0}$ for each $j=1, \cdots, k$ and assume that $Q_{j}=a_{m_{j}}^{j} X^{m_{j}}+a_{m_{j}-1}^{j} X^{m_{j}-1}+$ $\cdots+a_{0}^{j}, j=1, \cdots, k$. Now consider $\left\{a_{q}^{j}\right\}_{q=0,1, \cdots, m_{j}}^{j=1,2, \cdots, k}$, since this family is contained in $A$ and $C(J)$ is an essential left ideal in $A$, we can find an essential left ideal $K$ in $A$ such that $0 \neq K a_{q}^{j} \subseteq C(J)$ for each $j=1,2, \cdots, k, q=0,1, \cdots, m_{j}$. Hence $0 \neq K a_{q}^{j} \subseteq C_{s}(J)$ for some $s \in N$, which implies the existance of $\beta_{j} \in A$ such that $0 \neq \beta_{j} Q_{j} \in\left(C_{s}(J)\right)[X], j=$ $1, \cdots, k$. Let $\beta_{j} Q_{j}=\sum_{q=0}^{m_{j}} b_{q}^{j} X^{q}$ with at least one element of $b_{q}^{j} \neq 0$, denote it by $d_{j}$, as happend before in proposition 3.12 we can find an essential left ideal $L$ in $A$ such that $L b_{q}^{j} \subseteq \oplus_{i_{s} \in I_{s}} A f_{i_{s}}$ and $0 \neq L d_{j}$ for each $j=1,2, \cdots, k$. If $K_{j}$ is the complement of $\ell_{A}\left(d_{j} f_{t_{j}}\right)$ in $A$, then $K_{j} \cap L \neq 0$, which implies the existance of $\gamma_{j} \in K_{j} \cap L$ such that $0 \neq \gamma_{j}\left(d_{j} f_{t_{j}}\right)$. Therefore $0 \neq \gamma_{j}\left(\beta_{j} Q_{j}\right) \in\left(\oplus_{i_{s} \in I_{s}} A f_{i_{s}}\right)[X]$ and $\left(\gamma_{j}\left(\beta_{j} Q_{j}\right)\right) P_{t_{j}} \neq 0$ for each $j=1,2, \cdots, k$. But we have that $f_{i_{n}} \cdot f_{n}=f_{i_{n}}$ and $f_{i_{j}} f_{n}=0, j \neq n$, moreover $f_{i_{n}} a_{n}^{j}=f_{i_{n}} a_{i_{n}}^{j}$ for each $i_{j} \in \cup_{n} I_{n}$. Therefore $\left(\gamma_{j} \beta_{j} Q_{j}\right) P_{t_{j}}=Q_{j}^{\prime} P_{i_{j}}, Q_{j}^{\prime} \in A[X]$ and $P_{i_{j}}$ is one of the generators of $J^{*}$, for each $j=1, \cdots, k$, which implies that $\gamma_{j} \beta_{j} Q \in J^{*}$ for each $j=1,2, \cdots, k$. Hence $J^{*} \leq_{e} J^{\prime}$.

Theorem 3.15. If $A$ is a regular left self-injective ring and $J$ is a left ideal in $A[X]$ such that $C(J)$ is an essential left ideal in $A$, then there exists a countably generated left ideal $J^{\prime}$ in $A[X]$ such that $C\left(J^{\prime}\right)$ is an essential left ideal in $A$ and if $J^{\prime}$ is an essential left ideal in $A[X]$, then $J$ is an essential left ideal in $A[X]$.

Problem 1. Is $J^{\prime}$ is an essential left ideal in $A[X]$.

Remark 3.16. Let $A$ be a regular left self-injective ring and $J$ be a left ideal of $A$. If the answer of problem 1 is affirmative, then $C(J)$ is an essential left ideal in $A$, implies that $J$ is an essential left ideal in $A[X]$.

Problem 2. Let $A$ be a regular left self-injective ring and $I, J$ be left ideals in $A[X]$ such that $I \simeq J$ as a left $A[X]$-modules. Is $C(I) \simeq C(J)$ as left $A$-modules.

$\mathbb{R}$ emark 3.17. Let $A$ be a regular left slef-injective ring. If the answer of problems 1 and 2 are affirmative, then every directly finite idempotent in $A$ is a directly finite idempotent in $E(A[X])$.

Since if $e$ is directly finite in $A$ and $e E(A[X]) e$ is directly infinite, then from theorem 3.10 there exist two left ideals $I$ and $J$ in $(e A e)[X]$ such that $I \simeq J, I \leq_{e}(e A e)[X]$ and $J \mathrm{Le}_{e}(e A e)[X]$ as left $(e A e)[X]$-modules, which implies that $C(I) \simeq C(J), C(I) \leq_{e}$ $e A e$ and $C(J) \Sigma_{e} e A e$ as a left $e A e$-modules, but $e A e$ is a left slef-injective, hence this is a contradiction with $e A e$ is a directly finite ring.

In particular for regular left self-injective ring $A, A$ is a directly finite ring if and only if $E(A[X])$ is a directly finite ring. 
Proposition 3.18. If $A$ is a regular left self-injective ring, then $A$ contains abelian idempotents if and only if $E(A[X])$ contains abelian idempotents.

Proof. Let $f \neq 0$ be an abelian idempotent in $E(A[X])$, hence $f E(A[X]) f$ is an abelian regular ring and $I=E(A[X]) f \cap A[X]$ is a nonzero left ideal in $A[X]$. Let $P \in I, P=e X^{n}+a_{n-1} X^{n-1}+\cdots+a_{0}$ with minimal length and $e=e^{2} \in A^{*}$, hence we have that $A[X] P \simeq A[X] e$, which implies that $E(A[X]) . e \simeq E(A[X] P) \subseteq E(A[X]) f$. Therefore $E(A[X]) e$ is an abelian regular ring, hence $e$ is an abelian idempotent in $A$. Conversely if $e$ is an abelian idempotent in $A$, then $e$ is an abelian idempotent in $E(A[X])$, by using (2.10) and (2.14).

Remark 3.19. Let $A$ be a regular left self-injective ring, if the answers of problems 1 and 2 are affirmative, then

(1) $A$ is of type II if and only if $E(A[X])$ is of type II.

(2) $A$ is of type III if and only if $E(A[X])$ is of type III.

(3) $A$ is a purely infinite ring if and only if $E(A[X])$ is a purely infinite ring.

\section{References}

[1] A. Cailleau et G Renault, "Sur l'envoloppe injective des anneaux semi-premiers a ideal singular nul," Journal of algebra 15, (1970), 133-141.

[2] K.R.Goodearl, Von Neumann regular rings, (Pitman, London, 1979).

[3] J.M.Goursaud, Sur les anneaux introduits par la notion de module projectif. Thèse Préséntée a l'université de poiters (1977). [4] M. Henriksen, "On a class of regular rings that are elementary divisor rings," Arch. der Math., 24,
(1973) 133-141. [5] R.E. Johnson, "Structure theory of faithful rings II. Restticted rings," Thons. Amer. Math. Soc.,
84, (1957), 523-544. [6] Ahmed A.M. Kamal, "Regular left self-injective rings of type I," Afrika Matematika, Journal of
the african mathematical union (to appear).

[7] Ahmed A.M. Kamal, "Semiprimeness of polynomial rings" (to appear).

[8] I. Kaplansky, Rings of operators (Benjamin, New York, 1968).

[9] G. Renault, "Anneaux reguliers auta-injectifs a droite," Bull. Sci. France, 101, (1973) $237-254$.
[10] Y. Utumi, "On Continuous rings and self-injective rings," Trans. American Math. Soc. 118 (1965).

Mathematics Department, Faculty of Science, Cairo University, Giza, Egypt. 